\title{
SUR LES SOUS-ENSEMBLES BIEN ORDONNÉS DU CONTINU.
}

\author{
Par M. Johannes Mollerup (Kóbenhavn).
}

Adunanza del 14 aprile 1907.

M. G. Cantor a démontré (Journal de Crelle, vol. LXXVII) que le continu, c'est-ḋ-dire l'ensemble des nombres réels compris entre o et $\mathrm{I}$, n'est pas dénombrable. La puissance du continu est donc supérieure à celle de l'ensemble dénombrable. Par conséquent le contenu contient des sous-ensembles bien ordonnés de la deuxième puissance, c'est-d̀-dire des sous-ensembles

$$
a_{1}, a_{2}, a_{3}, \ldots, a_{\omega}, \ldots, a_{\Upsilon}, \ldots,
$$

ou $\gamma$ parcourt tous les nombres de la deuxième classe de nombres de M. Cantor. Je vais faire dans ce qui suit une recherche de la structure de tels sous-ensembles.

Défrnition. - Une série fondamentale de la deuxième espèce est un ensemble bien ordonné des nombres réels de la puissance de la deuxième classe de nombres

$$
a_{1}, a_{2}, a_{j}, \ldots, a_{\omega}, \ldots, a_{\Upsilon}, \ldots
$$

jouissant de la propriété suivante:

soit $\varepsilon$ un nombre positif donné; alors il existe un nombre $\gamma$ de la première ou de la deuxième classe tel que la relation

$$
\left|a_{\gamma_{1}}-a_{\gamma_{2}}\right|<\varepsilon
$$

sera satisfaite toutes les fois que $\gamma_{\mathrm{r}}$ et $\gamma_{2} \supseteq \gamma$.

Suivant le théorème de Bolzano-Weierstrass sur l'existence des points-limites, chaque sous-ensemble infini d'une telle série fondamentale de la deuxième espèce a au moins un point limite; il faut qu'on ne confonde pas ces points-limites avec le pointlimite particulier que je vais mentionner dans le théorème suivant.

Théoréme I. - Une série fondamentale de la deuxième espèce

$$
a_{1}, a_{2}, a_{3}, \ldots, a_{\omega}, \ldots, a_{r}, \ldots
$$

a un point-limite particulier jouissant de la proprieté suivante:

soit donné un intervalle autour de a, il existe un nombre $\gamma$ de la premiere ou de la deuxième classe de nombres tel que tous les a dont les indices sont plus grands que, ou égaux $\dot{a}, \gamma$, se trouvent dans l'intervalle. 
Soit

$$
\varepsilon_{\mathrm{r}}>\varepsilon_{2}>\varepsilon_{3}>\cdots
$$

une série fondamentale de nombres positifs décroissants, ayant pour limite o. A ces nombres correspondent des nombres de la première on de la deuxième classe:

$$
r_{1} \leq \gamma_{2} \leq \gamma_{3} \leq \cdots \quad \text { (suivant la définition précédente). }
$$

Suivant le théorème sur l'existence des points-limites, chacun des ensembles dénombrables

$$
\begin{aligned}
& a_{\Upsilon_{1}}, \quad a_{\Upsilon_{I}+1}, \quad a_{\gamma_{1}+2}, \ldots \\
& a_{\Upsilon_{2}}, \quad a_{\Upsilon_{2}+1}, \quad a_{\gamma_{2}+2}, \ldots \\
& a_{\gamma_{\nu}}, a_{\gamma_{\nu}+1}, a_{\gamma_{\nu}+2}, \ldots \\
& \ldots \ldots \ldots \ldots
\end{aligned}
$$

contient une série fondamentale; soient celles-ci

$$
\begin{aligned}
& a_{\Upsilon_{11}}, \quad a_{r_{12}}, a_{r_{1}}, \ldots \\
& a_{\Upsilon_{21}}, a_{\gamma_{22}}, a_{\Upsilon_{23}}, \ldots \\
& \ldots \ldots \ldots \\
& a_{\gamma_{v 1}}, a_{\gamma_{v 2}}, a_{\gamma_{v_{3}}}, \ldots
\end{aligned}
$$

ayant les points-limites

L'ensemble

$$
b_{\gamma_{1}}, \quad b_{\gamma_{2}}, b_{\gamma_{\nu}}, \ldots
$$

contient une série fondamentale ayant le point-limite $b_{\gamma}$.

$$
b_{Y_{1}}, \quad b_{\gamma_{2}}, b_{Y_{\nu}}, \ldots
$$

$$
b_{\gamma_{\nu_{1}}}, b_{\gamma_{\nu_{2}}}, \ldots
$$

On a ici

$$
\begin{aligned}
& b_{\gamma_{\nu_{1}}}=\lim \left(a_{\gamma_{v_{1}}}, \quad a_{\left.\gamma_{v_{1}}, \ldots\right)},\right. \\
& b_{r_{v_{2}}}=\lim \left(a_{r_{v_{2}} \mathrm{I}}, \quad a_{r_{v_{2}}{ }^{2}}, \ldots\right) \text {, }
\end{aligned}
$$

Maintenant je vais démontrer que $b_{\Upsilon}$ sera le point-limite particulier de la série fondamentale de la deuxième espèce (il est clair qu'il n'existe pas plusieurs points-limites particuliers).

Soit $\varepsilon>0$ un nombre donné; on pourra déterminer un entier $\mu$ tel que l'on ait

$$
\varepsilon_{\mu} \leq \frac{\varepsilon}{3}<\varepsilon_{\mu-1} .
$$

Alors on détermine $m$ tel que l'on ait

$$
\left|b_{\Upsilon}-b_{\gamma_{v_{m}}}\right|<\frac{\varepsilon}{3} \quad \text { et } \quad v_{m}>\mu,
$$

et enfin on détermine $n$ et $p$ tels que l'on ait

$$
\left|b_{\gamma_{v_{m}}}-a_{\gamma_{v_{m}}}\right|<\frac{\varepsilon}{3} \text { et } \mu>\gamma_{\mu} \text {. }
$$


On aura donc

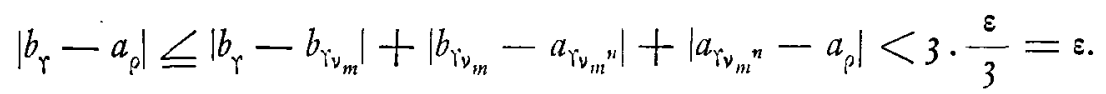

Alors $b_{r}$ sera le point-limite particulier cherché.

ThÉorème II. - Soit $b_{r}$ le point-limite particulier d'une série fondamentale de la deuxième espèce

$$
a_{1}, a_{2}, a_{j}, \ldots, a_{\omega}, \ldots, a_{\gamma}, \ldots \text {; }
$$

il existe alors, à l'interieur de celle-ci, une série fondamentale ordinaire ayant pour pointlimite $b_{r}$.

Pour l'ensemble

on détermine $\lambda_{1}$ tel que l'on ait

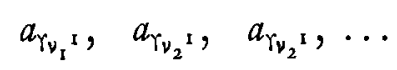

pour l'ensemble

$$
\left|a_{\gamma_{v_{1}} \lambda_{1}}-b_{\gamma_{v_{1}}}\right|<\varepsilon_{\mathrm{r}}
$$

on détermine $\lambda_{2}$ tel que l'on ait

$$
a_{r_{v_{2} 1}}, a_{r_{v_{2}}}, \ldots
$$

et ainsi de suite.

$$
\left|a_{\gamma_{v_{2}} \lambda_{2}}-b_{\gamma_{v_{2}}}\right|<\varepsilon_{2}
$$

On a donc

L'ensemble

$$
\left|a_{\Upsilon_{v_{m} \lambda_{m}}}-b_{\Upsilon_{\nu_{m}}}\right|<\varepsilon_{m}
$$

$$
a_{\gamma_{1} \lambda_{1}}, a_{\gamma_{\nu_{2}} \lambda_{2}}, \ldots
$$

sera alors une série fondamentale ordinaire ayant le point-limite $b_{r}$.

ThÉorìme III. - Soit $b_{\Upsilon}$ le point-limite particulier d'une série fondamentale de la deuxième espèce

$$
a_{1}, a_{2}, \ldots, a_{\omega}, \ldots, a_{\Upsilon}, \ldots \text {; }
$$

il existe alors un entier $;$ de la première on de la deuxième classe de sorte que l'on a constamment, des que $\sigma \bar{\gamma} \rho$,

$$
a_{\tau}=b_{r} .
$$

En effet, on trouvera dans la deuxième classe un nombre $\rho$ plus grand que chaque nombre de l'ensemble

$$
\gamma_{\nu_{1} \lambda_{1}}, \quad \gamma_{v_{2} \lambda_{2}}, \quad \gamma_{v_{3} \lambda_{3}}, \ldots
$$

puisque dans cette classe chaque ensemble bien ordonné dénombrable est suivi d'un nombre nouveau.

Alors on aura, $\gamma_{v_{m} \lambda_{m}}$ et $\rho$ étant tous les deux $>m$,

$$
\left|\dot{a}_{\rho}-b_{\gamma_{v_{m}}}\right| ₹\left|b_{i_{v_{m}}}-a_{i_{v_{m} \lambda_{m}}}\right|+\left|a_{\gamma_{v_{m}} \lambda_{m}}-a_{\rho}\right|<2 \varepsilon_{m} .
$$

Cette différence convergera vers zéro, $b_{\gamma_{v_{m}}}$ parcourant la série fondamentale

$$
b_{i v_{1}}, b_{i v_{2}}, \ldots
$$

Alors $a_{\rho}$ sera le point-limite de cette série et coïncidera avec $b_{\gamma} \cdot \rho$ est ici un entier arbitraire de la deuxième classe, plus grand que tous les nombres

$$
\gamma_{v_{1} \lambda_{1}}, \gamma_{v_{2} \lambda_{2}}, \ldots
$$


De ces théorèmes on voit que des séries fondamentales de la deuxième espèce ì proprement parler n'existent pas: dès que l'indice du terme sera devenu assez grand, tous les termes seront identiques. L'ensemble de tous les nombres qui précèdent un nombre donné de la deuxième classe étant dénombrable, le sous-ensemble des termes différents entre eux sera dénombrable.

THĖorème IV. - Étant donné un sous-ensemble bien ordonné du continu, ayant la deuxième puissance,

$$
a_{1}, a_{2}, a_{3}, \ldots, a_{\omega}, \ldots, a_{\gamma}, \ldots,
$$

il existe un nombre reel $\varepsilon, 0<\varepsilon<\mathrm{I}$, tel que

$$
\left|a_{\rho_{1}}-a_{\rho_{2}}\right|<\varepsilon
$$

$\rho_{1}$ et $\rho_{2}$ étant plus grands qu'un nombre déterminé $\rho$; d'autre part, $\varepsilon^{\prime}$ étant $<\varepsilon$ et $\left|a_{\rho_{\mathrm{r}}}-a_{\rho_{2}}\right|<\varepsilon^{\prime}$ pour certaines valeurs de $\rho_{1}$ et $\rho_{2}$, cette différence sera $>\varepsilon^{\prime}$ pour des valeurs plus grandes de $p_{1}$ et $p_{2}$.

En effet, l'ensemble

$$
a_{1}, a_{2}, \ldots, a_{\omega}, \ldots, a_{r}, \ldots
$$

n'étant pas une série fondamentale, il existe un nombre réel positif $\Sigma_{1}$, tel que, $\left|a_{\rho_{1}}-a_{p_{2}}\right|$ étant $\left\langle\varepsilon_{1}\right.$, il y a toujours des indices plus grands tels que cette différence soit $>\varepsilon_{1}$. Soient $n_{1}, n_{2}, \ldots, n_{m}, \ldots$ une suite de nombres positifs décroissants ayant $\varepsilon_{1}$ pour limite; alors on ne pourra pas pour chaque valeur de $v$ déterminer un entier $\rho$ tel que l'on ait constamment, dès que $\sigma$ et $\tau>\rho$,

$$
\left|a_{\sigma}-a_{\tau}\right|<n_{y} .
$$

Supposons en effet, que la suite

correspond à la suite

$$
p_{1}, p_{2}, p_{3}, \ldots
$$

de sorte que l'on ait

$$
\begin{gathered}
n_{1}, n_{2}, n_{3}, \ldots, \\
\left|a_{\sigma}-a_{\tau}\right|<n_{v}
\end{gathered}
$$

dès que $\sigma$ et $\tau>\rho_{v}$; on pourra déterminer un entier $\rho$ plus grand que tous les nombres

$$
p_{1}, p_{2}, p_{3}, \ldots
$$

Alors la différence $\left|a_{\sigma}-a_{\tau}\right|$ sera plus petite que tous les nombres $n_{1}, n_{2}, n_{3}, \ldots$, dès que $\sigma$ et $\tau$ sont $>\rho$, et par suite $\left|a_{\sigma}-a_{\tau}\right|=\varepsilon_{1}$, ce qui est impossible. Alors il faut qu'il existe un nombre $n_{y}$ tel que, $\left|a_{f_{1}}-a_{\rho_{2}}\right|$ étant $<r_{y}$, on pourra trouver des indices supérieurs, pour lesquels la différence sera $>n_{\nu}$. Il est donc démontré que, $\varepsilon_{\mathrm{I}}$ étant un nombre réel et positif comme au commencement de la démonstration, il existera toujours un nombre plus grand ayant la même propriété.

Le nombre I étant tel que

$$
\left|a_{\rho_{1}}-a_{\rho_{2}}\right|<\mathrm{I}
$$


le sous-ensemble donné du continu déterminera une coupure de DeDekind des nombres réels entre $O$ et I; cette coupure nous fera connaitre le nombre cherché $\varepsilon$.

Théorème V. - Un sous-ensemble bien ordonné du continu de la deuxième puissance

$$
a_{1}, a_{2}, \ldots, a_{\omega}, \ldots, a_{r}, \ldots
$$

déterminera un intervalle $(k l), 0 \leq k<l \leq \mathrm{I}$, contenant tous les derniers elléments, c'est-à-dire tous les éléments dont les indices sont plus grands qu'un nombre $\rho$; d'autre part, aucun sous-intervalle de $(k l)$ ne contiendra tous les derniers éléments, c'est-à-dire, étant donné un sous-intervalle de $(k l)$ il n'existe jamais un nombre $p$ tel que tous les éléments des indices $>\rho$ soient situés dans ce sous-intervalle. Ainsi l'intervalle $(k l)$ sera particulièrement représenté dans l'ensemble donné.

Tout le sous-ensemble donné est contenu dans l'intervalle (O I); d'autre part, il existe un intervalle (os) qui ne contient pas tous les derniers éléments (Th. IV). Il est aussi démontré qu'il existe toujours un intervalle $\left(o \varepsilon_{1}\right), \varepsilon_{1}>\varepsilon$, ne contenant pas tous les derniers éléments. Alors, l'ensemble donné fera une coupure des nombres réels, qui déterminera un nombre $l \neq \mathrm{r}$, tel que l'intervalle $(\mathrm{o} l$ ) contiendra tous les derniers éléments, tout intervalle $\left(\mathrm{o} l_{\mathrm{r}}\right), l_{1}<l$ ne contenant pas tous les derniers éléments. Également il existe un intervalle $(k \mathrm{I}), o \leq k<\mathrm{I}$, contenant tous les derniers éléments; il faut que $k<l$. L'intervalle $(k l)$ contiendra donc tous les derniers éléments.

Par conséquent, chaque intervalle $(k a), k<a$, et chaque intervalle $(l b), b<l$, contiendront un sous-ensemble de l'ensemble donné, ayant la deuxieme puissance; car, supposant qu'un intervalle $(k a)$ ne contienne qu'un sous-ensemble dénombrable, on voit que tous les derniers éléments se trouvent dans l'intervalle $(a l)$, ce qui est impossible.

ThÉorème VI. - Un sous-ensemble bien ordonné du continu de la deuxième puissance

$$
a_{\mathrm{r}}, a_{2}, \ldots, a_{\omega}, \ldots, a_{r}, \ldots
$$

s'il n'a pas dans chaque sous-intervalle de l'intervalle $(k l) d u$ Théoreme $V$ un sous-ensemble de la deuxième puissance, il determinera dans cet intervalle une série de sous-intervalles correspondants aux entiers positifs et négatifs

oì

$$
\ldots\left(b_{-n} a_{-n}\right) \ldots\left(b_{-2} a_{-2}\right)\left(b_{-1} a_{-1}\right)\left(b_{0} a_{0}\right)\left(b_{1} a_{1}\right)\left(b_{2} a_{2}\right) \ldots\left(b_{n} a_{n}\right) \ldots
$$

$$
k<b_{-n}<a_{-n} \cdots<b_{0}<a_{0}<b_{1}<a_{1}<\cdots<a_{n} \cdots<l,
$$

de sorte que chaque intervalle ne contient qu'un ensemble dénombrable des eléments de l'ensemble donné. D'autre part, chaque intervalle

$$
\ldots\left(a_{-n} b_{-(n-1)}\right) \ldots\left(a_{-2} b_{-1}\right)\left(a_{-1} b_{0}\right)\left(a_{0} b_{1}\right)\left(a_{1} b_{2}\right) \ldots\left(a_{n} b_{n+1}\right) \ldots
$$

contiendra un sous-ensemble de la deuxieme puissance. Dans chaque intervalle arbitrairement petit à droite d'un terme a et à gauche d'un terme $b$ il se trouve un sous-ensemble de la deuxième puissance. Si la série d'intervalles est fini à droite ou à gauche, il existe un intervalle tel que chaque sous-intervalle contienne un sous-ensemble de la deuxième puissance.

En supposant qu'il y ait des sous-intervalles de $(k l)$ qui ne contiennent pas un sousensemble de l'ensemble donné ayant la deuxième puissance, il y a un point $a_{\mathrm{o}}$ ayant 
un sous-ensemble dénombrable dans un intervalle à gauche. Le théorème précédent déterminera donc $b_{\mathrm{o}}$ tel que $b_{\mathrm{o}}$ ait un sous-ensemble de la deuxième puissance dans chaque intervalle à gauche. $a_{-1}$ et $b_{-1}$ seront également déterminés, etc.; la série ne finira qu'en arrivant $\mathrm{a}$ un intervalle $\left(k b_{-n}\right)$ dont chaque sous-intervalle contient un ensemble de la deuxième puissance. Selon le théorème précídent, on peut déplacer dans l'intervalle $\left(b_{-n} b_{-(n-1)}\right) a_{-n}$ à droite si loin que possible, $\left(b_{-n} a_{-n}\right)$ ne contenant toujours qu'un ensemble dénombrable, $\left(a_{-n} b_{-(n-1)}\right)$ contenant au contraire un ensemble de la deuxième puissance.

De la même manière, le sous-ensemble contenu dans l'intervalle $\left(l a_{0}\right)$ déterminera un point à droite de $a_{\mathrm{o}}$, où on peut placer $a_{\mathrm{o}}$ de manière que $\left(l a_{\mathrm{o}}\right)$ contient tous les derniers ćléments du sous-ensemble nommé, aucun sous-intervalle ne contenant les derniers éléments. S'il y a dans $\left(a_{0} l\right)$ un point $b_{\mathrm{s}}$ ayant un sous-ensemble dénombrable dans un intervalle a droite, on déterminera dans $\left(b_{1} l\right)$ le point $a_{\mathrm{r}}$ tel que $\left(b_{1} a_{1}\right)$ contienne un sous-ensemble dénombrable, $a$, ayant un sous-ensemble de la deuxième puissance dans chaque intervalle id droite. Ainsi continuera-t-on, et enfin $b_{1}, b_{2}, \ldots$ sont déplacés à gauche si loin que possible, $\left(b_{1} a_{1}, b_{2} a_{2}, \ldots\right)$ ne contenant toujours qu'un ensemble dénombrable. Aucun intervalle ne peut disparaitre; mais on peut finir à droite avec un $a$ et à gauche avec un $b$.

S’il n'y a pas d'intervalle qui ait la propriété de contenir partout un sous-ensemble de la deuxième puissance, nous nous pourrons assurer que la série $b_{0}, b_{-1}, b_{-2}, \ldots$ converge vers $k$, en faisant converger vers $k$ la série des $a$ déterminés les premiers. Si la série des $a$ déplacés converge vers un nombre $A>k$, un intervalle du voisinage de $k$ jusqu'à $A$ ne contiendrait jamais qu'un ensemble dénombrable, ce qui est impossible.

Donc la série des a déplacés convergera aussi vers $k$. De la même manière les intervalles $\left(b_{n} a_{n}\right)$ convergent vers $l$.

ThÉorème VII. - Étant donné un sous-ensemble bien ordonné du continu de la deuxieme puissance

$$
a_{1}, a_{2}, a_{3}, \ldots, a_{\omega}, \ldots a_{Y}, \ldots,
$$

il existera toujours dans l'intervalle $(k l)$ correspondant des points $w$ de la propriété que l'ensemble donné ait un sous-ensemble de la deuxième puissance et dans chaque intervalle à droite et dans chaque intervalle à gauche de $w$.

S'il se trouve un intervalle dans $(k l)$ dont chaque sous-intervalle contient un sousensemble de l'ensemble donné, ayant la deuxième puissance, le théorème sera trivial.

Dans le cas contraire nous ferons la division d'intervalles du théorème précédent; alors les intervalles $\left(a_{n} b_{n+1}\right), n$ étant un entier positif ou négatif, contiennent toujours des sous-ensembles de la deuxiène puissance. Si l'on traite ces intervalles de la même manièrè, il s'établira des sous-intervalles $\left(a_{n_{1} n_{2}}, b_{n_{1}+1, n_{2}+1}\right), n_{1}$ et $n_{2}$ étant des entiers arbitraires, positifs ou négatifs, contenant toujours des sous-ensembles de la deuxième puissance. En continuant de la sorte, on trouvera une série d'intervalles

$$
\left(a_{n_{1}} b_{n_{1}+1}\right)\left(a_{n_{1} n_{2}} b_{n_{1}+1, n_{2}+1}\right) \ldots\left(a_{n_{1} n_{2} \ldots n_{p}} b_{n_{1}+i_{1} n_{2}+1, \ldots n_{p}+1}\right) \ldots \text {, }
$$

chaque intervalle étant contenu dans le précédent; cette série convergera vers un pointlimite $w$ ayant la proprièté cherchée. 
Si la puissance du continu est supérieure à celle de la deuxième classe de nombres, les théorèmes précédents resteront vrais pour des sous-ensembles bien ordonnés des puissances suivantes.

Si l'on accepte le théorème de M. Zermelo (Math. Ann., t. LIX, page 5I4), la puissance du continu sera un "aleph»; en ce cas les théorèmes précédents seront tous triviaux pour le continu lui-même, tandis qu'ils ne le seront point pour les sous-ensembles bien ordonnés. Il me semble que le Théorème III conserve l'importance au delà des recherches sur le problème du continu dont la solution d'une manière naturelle est à chercher par une étude approfondie des sous-ensembles bien ordonnés.

Copenhague, mars 1907.

Johannes Mollerup. 